Keynote address

\title{
The new norm and a changed role for the student
}

\author{
Harden, R.M.
}

General Secretary, Association for Medical Education in Europe (AMEE)

Increased student engagement in the education programme is an important development in medical education and this has been given an added momentum by the COVID-19 pandemic.

On today's agenda is the shift from the student as a customer and a passive recipient of the teaching and education offering to one of a student engaged as a partner in the programme. The implications of this move in practice are at times difficult and at times uncertain. Ambiguities can be clarified by considering the roles of the student in the engagement process. Seven roles can be identified.

-Information processor and seeker

-Facilitator of learning

-Curriculum collaborator

-Assessor of competence

-Teacher and mentor

-Professional

-Manager

The roles are reflected in the different approaches to student engagement, in the extent to which students have a voice in decision making, and in the nature of a student/teacher partnership. 